\title{
Novel PYGL mutations in Chinese children leading to glycogen storage disease type Vl: two case reports
}

\author{
Xiaomei Luo ${ }^{1,2}$, Jiacheng $\mathrm{Hu}^{3}$, Xueren Gao ${ }^{1,2}$, Yanjie Fan ${ }^{1,2}$, Yu Sun ${ }^{1,2}$, Xuefan $\mathrm{Gu}^{1,2}$ and Wenjuan Qiu ${ }^{1,2^{*}}$
}

\begin{abstract}
Background: PYGL mutations can cause liver phosphorylase deficiency, resulting in a glycogenolysis disorder, namely, glycogen storage disease (GSD) VI. The disease is rarely reported in the Chinese population. GSD VI is mainly characterized in untreated children by hepatomegaly, growth retardation and elevated liver transaminases.

Case presentation: In this study, we report two GSD VI patients with growth retardation and abnormal liver function. There was no obvious hepatomegaly for one of them. Whole exome sequencing (WES) combined with copy number variation analysis was performed. We found a novel homozygous gross deletion, c.1621-258_2178-23del, including exons 14-17 of PYGL in patient 1. The exons 14-17 deletion of PYGL resulted in an in-frame deletion of 186 amino acids. Compound heterozygous mutations of PYGL were identified in patient 2, including a novel missense mutation c.1832C > T/p.A611V and a recurrent nonsense mutation c.280C > T/p.R94X. After treatment with uncooked cornstarch (UCS) 8 months for patient 1 and 13 months for patient 2, the liver transaminases of both patients decreased to a normal range and their stature was improved. However, patient 1 still showed mild hypertriglyceridemia.

Conclusions: We describe two GSD VI patients and expand the spectrum of PYGL mutations. Patient 1 in this study is the first GSD VI case that showed increased transaminases without obvious hepatomegaly due to a novel homozygous gross deletion of PYGL identified through WES.
\end{abstract}

Keywords: Glycogen storage disease VI, Inherited metabolic disease, Molecular diagnosis, Whole exome sequencing

\section{Background}

Glycogen storage diseases (GSD) are a group of inherited metabolic disorders characterized by abnormal intracellular accumulation of glycogen $[1,2]$. Overall, the incidence of GSD is estimated to be 1 case per 20,000 to 43,000 live births. There are over 12 types of GSD, classified based on the enzyme deficiency and the affected tissue [2]. Glycogen storage disease VI (GSD VI; Hers disease; OMIM 232700) is a rare form of GSD caused by deficiency of liver phosphorylase due to compound heterozygous or

\footnotetext{
* Correspondence: qiuwenjuan@xinhuamed.com.cn

'Department of Pediatric Endocrinology/Genetics, Xinhua Hospital, Shanghai Jiao Tong University School of Medicine, Shanghai 200092, China

${ }^{2}$ Shanghai Institute for Pediatric Research, Shanghai 200092, China

Full list of author information is available at the end of the article
}

homozygous mutations of PYGL [3]. The clinical manifestations of GSD VI are similar to other forms of GSD associated with the liver, such as GSD I, GSD III, GSD IXa and GSD XI, while the treatment may be different. GSD VI is characterized in untreated children by hepatomegaly, growth retardation, and mild hypoglycemia after overnight fasting. This disease is usually a relatively mild disorder that presents in infancy and childhood. The PYGL gene is located on chromosome 14q22.1 and has 20 exons in total. Currently, the Human Gene Mutation Database (http://www.hgmd.cf.ac.uk/ac/index.php) has reported around 50 mutations in PYGL associated with GSD VI. In this study, we describe two unrelated Chinese GSD VI patients. One case resulted from a PYGL homozygous large fragment deletion that was identified through copy

(c) The Author(s). 2020 Open Access This article is licensed under a Creative Commons Attribution 4.0 International License, which permits use, sharing, adaptation, distribution and reproduction in any medium or format, as long as you give appropriate credit to the original author(s) and the source, provide a link to the Creative Commons licence, and indicate if changes were made. The images or other third party material in this article are included in the article's Creative Commons licence, unless indicated otherwise in a credit line to the material. If material is not included in the article's Creative Commons licence and your intended use is not permitted by statutory regulation or exceeds the permitted use, you will need to obtain permission directly from the copyright holder. To view a copy of this licence, visit http://creativecommons.org/licenses/by/4.0/ The Creative Commons Public Domain Dedication waiver (http://creativecommons.org/publicdomain/zero/1.0/) applies to the data made available in this article, unless otherwise stated in a credit line to the data. 
number variations analysis of WES depth data and the other case resulted from PYGL compound heterozygous point mutations. Interestingly, the patient with the gross deletion showed elevated liver transaminases without obvious hepatomegaly, which was unusual in GSD patients. This study was approved by Xinhua Hospital Ethics Committee Affiliated to Shanghai Jiaotong University School of Medicine (XHEC-D-2019-057).

\section{Case presentation}

\section{Case 1}

A 17-month-old Chinese girl was referred to our hospital due to unexplained elevated liver transaminases. She was the third child of consanguineous parents, was delivered at full term and weighed $3350 \mathrm{~g}$. There was no family history of liver disease. Physical examination showed growth retardation with a height of $72 \mathrm{~cm}(-2.87 \mathrm{SD})$ and a weight of $9 \mathrm{~kg}$. The height of her father is $155 \mathrm{~cm}(-2.90 \mathrm{SD})$ and that of her mother is $157 \mathrm{~cm}(-0.67 \mathrm{SD})$. There was no obvious mental or motor developmental delay. The patient had no obvious hepatomegaly or splenomegaly. Laboratory examinations in the local hospital showed increased liver enzymes, lactate, and triglycerides after fasting. Her blood glucose was normal or occasionally reduced after different fasting periods.

Whole exome sequencing (WES) was applied to detect small deletions/insertions and single nucleotide variations. The exome library was constructed using an xGen Exome Research Panel v1.0 (Integrated DNA Technologies, San Diego, CA, USA). An Illumina Hi-seq 4000 sequencer (Illumina, San Diego, CA, USA) was used for high-throughput sequencing. Variants were filtered based on frequency, inheritance pattern, clinical phenotype and pathogenicity.

The variant calling software revealed a large fragment deletion of the PYGL gene in patient 1. The Integrative Genomics Viewer showed this homozygous c.1621-258 2178-23del through the bam file (Fig. 1a), which was a gross in-frame deletion. The deletion, which included exons 14-17, was 3581 bp and located on chr14 nt. 51, $375,696-51,379,279$. The fragments (exons 14 to 17) of the parents could be amplified, while the products of the

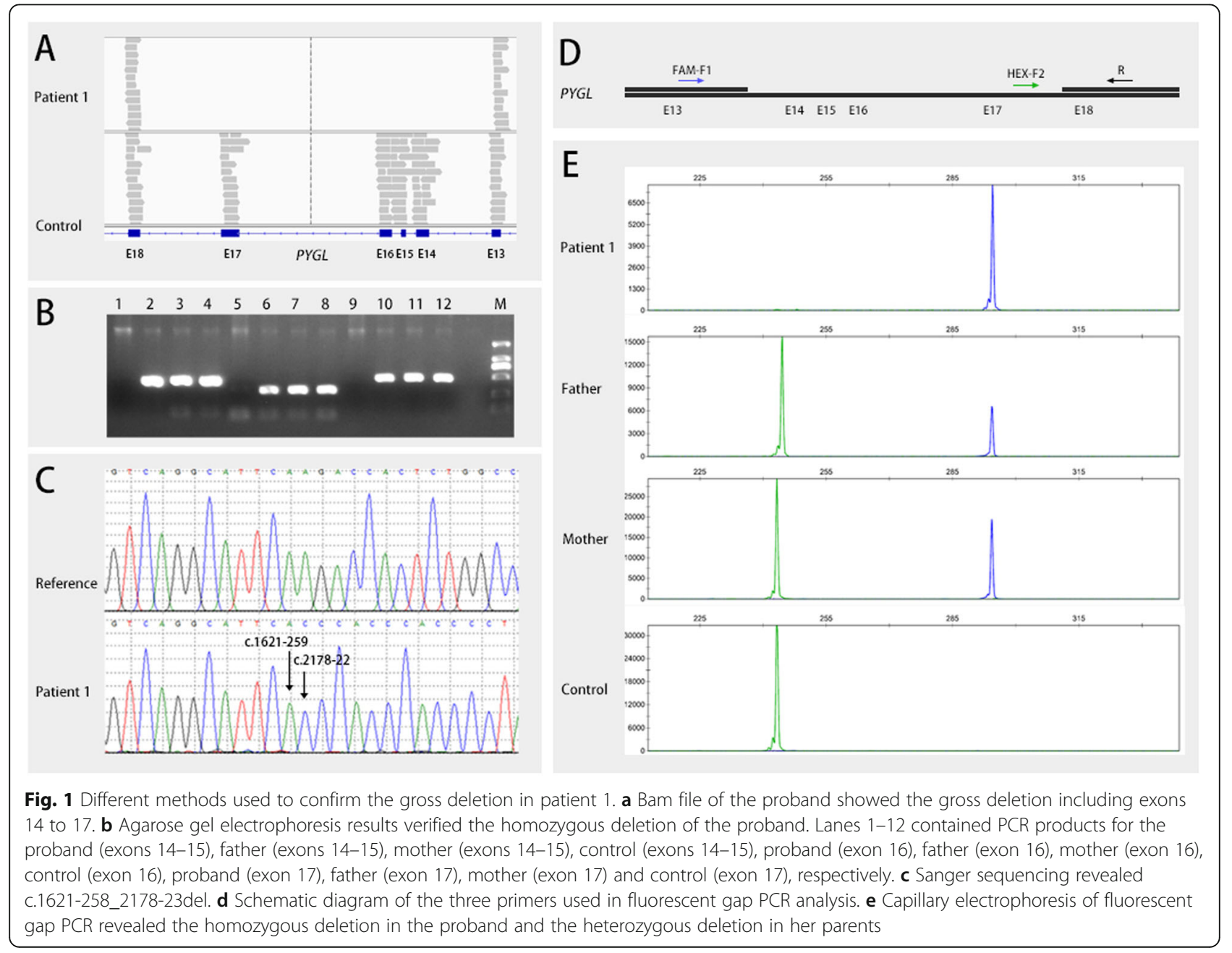


proband were absent according to the agarose gel electrophoresis (Fig. 1b). Sanger sequencing was performed to verify the breakpoints of the deletion, which was NM 002863.4: c.1621-258_2178-23del (Fig. 1c). The reference sequence in Fig. 1c was from NCBI reference sequences NG_012796.1. Fluorescent gap PCR analysis was used to verify the deletion. The primers F and R2 flank the PYGL deletion, whereas the primer R1 anneals within the deleted region (Fig. 1d). The capillary electrophoresis results of fluorescent gap PCR products confirmed the homozygous deletion in the proband and revealed that the parents were both heterozygous deletion carriers (Fig. 1e). The primers used for PCR amplification are listed in Supplementary materials (Table S1, S2 and S3).

Uncooked cornstarch (UCS) 4 times a day $(1.5 \mathrm{~g} / \mathrm{kg} /$ each time) was recommended for treatment. Patient 1 improved her growth from -2.87 SD to -1.96 SD after 8 months of treatment, and all the biochemical parameters except for triglycerides were normal. Her triglycerides value decreased from $4.39 \mathrm{mmol} / \mathrm{L}$ to $2.15 \mathrm{mmol} / \mathrm{L}$ after treatment for 8 months, while it was still slightly above the normal range $(0.56-1.69 \mathrm{mmol} / \mathrm{L})$. The detailed laboratory values at diagnosis and after treatment for patient 1 are listed in Table 1.

\section{Case 2}

This patient was a 26-month-old Chinese girl who was the second child of nonconsanguineous parents. The child was born after a normal gestational period. There

Table 1 Biochemical parameters of the two patients before and after treatment

\begin{tabular}{|c|c|c|c|c|c|}
\hline & \multicolumn{2}{|l|}{ Patient 1} & \multicolumn{2}{|c|}{ Patient 2} & \multirow[t]{2}{*}{ Reference } \\
\hline & Before & After $^{\mathrm{a}}$ & Before & After $^{b}$ & \\
\hline Glucose (mmol/L) & 4.26 & 4.23 & 3.43 & 4.2 & $3.8-6.2$ \\
\hline $\operatorname{ALT}(U / L)$ & 162.8 & 30 & 92 & 11 & $10-40$ \\
\hline AST (U/L) & 230.9 & 38 & 102 & 28 & $10-40$ \\
\hline$\gamma G T(U / L)$ & 61 & 10 & 74 & 11 & $0-25$ \\
\hline Lactate (mmol/L) & $4.03 / 2.5^{c}$ & 1.0 & NA & 1.3 & $0.7-2.1$ \\
\hline TG (mmol/L) & 4.39 & 2.15 & 4.37 & 1.57 & $0.56-1.69$ \\
\hline TC (mmol/L) & 3.47 & 3.09 & 3.7 & 3.76 & $2.33-5.69$ \\
\hline Uric acid ( $\mu \mathrm{mol} / \mathrm{L})$ & 342 & 208 & 402 & 322 & $100-410$ \\
\hline Total protein (g/L) & 58.2 & 60.1 & 66.4 & 70.7 & $58-80$ \\
\hline Prealbumin (mg/L) & 180 & NA & 160 & NA & $100-300$ \\
\hline Ketone $(\mathrm{mmol} / \mathrm{L})^{d}$ & $\mathrm{~N}$ & $\mathrm{~N}$ & $\mathrm{~N}$ & N & $\mathrm{N}$ \\
\hline CK & 22 & NA & 51 & NA & 18-173 \\
\hline
\end{tabular}

Abbreviations: ALT alanine transaminase, AST aspartate aminotransferase, $\gamma G T$ gamma-glutamyl transpeptidase, $T G$ triglycerides, $T C$ total cholesterol, $N$ negative, $N A$ not available

${ }^{\text {a }}$ The laboratory values of patient 1 after treatment for 8 months

${ }^{\mathrm{b}}$ The laboratory values of patient 2 after treatment for 13 months

'The lactate concentration had been tested for twice after fasting

${ }^{d}$ Ketone test used urine sample, and the other biochemical tests used serum samples was no family history of liver disease. She was admitted to the hospital due to growth retardation with a height of $83 \mathrm{~cm}(-1.67 \mathrm{SD})$. The height of her father is $176 \mathrm{~cm}$ $(+0.54 \mathrm{SD})$, and that of the mother is $164 \mathrm{~cm}(+0.63$ SD). Physical examination showed hepatomegaly $(7 \mathrm{~cm}$ below the right costal margin). Laboratory tests showed significantly increased liver transaminases, elevated triglycerides and slightly decreased glucose.

WES was performed as described above. The results suggested that patient 2 inherited compound heterozygous mutations on the PYGL gene, including c. $280 \mathrm{C}>\mathrm{T} /$ p.R94X on exon 2 from her mother and c.1832C $>\mathrm{T} /$ p.A611V on exon 16 from her father (Fig. 2). The missense mutation c. $1832 \mathrm{C}>\mathrm{T} / \mathrm{p} . \mathrm{A} 611 \mathrm{~V}$ is a novel mutation that had not been reported previously and can be classified as a likely pathogenic mutation (PM2 + PM3 + PP3 + PP4) according to the American College of Medical Genetics guidelines for variant interpretation [4].

The patient increased from $-1.67 \mathrm{SD}$ to $-1.61 \mathrm{SD}$ after 13 months of UCS treatment as patient 1 , and the size of her liver was normal. All her biochemical parameters were normal. The detailed laboratory values at diagnosis and after treatment for patient 2 are listed in Table 1.

\section{Discussion and conclusions}

GSD VI is caused by deficient liver phosphorylase activity, resulting in excessive accumulation of glycogen in the liver. The disease is usually a relatively mild disorder characterized in untreated children by hepatomegaly, growth retardation, mild hypoglycemia, and increased liver transaminases. Generally, several liver GSDs, such as GSD I, GSD III, GSD VI and GSD IX, often manifest with similar phenotypes such as short stature, hypoglycemia and hepatomegaly. GSD I patients are more severe without ketosis. GSD III infants usually have more obvious elevated liver enzyme and ketosis. GSD XI patients have malabsorption, renal abnormalities and acidosis. Among these different GSD types, GSD IXa is the type that is most similar to GSD VI because it results from deficiency of liver phosphorylase kinase, and phosphorylase kinase deficiency can itself lead to reduced liver phosphorylase activity [5].

Next-generation sequencing has been considered the preferred diagnostic method for GSD [5-7] because invasive liver biopsy can be avoided. We identified a novel homozygous gross deletion mutation (c.1621-258_217823del) of PYGL in patient 1 through WES. This gross deletion was further verified through agarose gel electrophoresis and fluorescent gap PCR analysis. For patient 2, we identified compound heterozygous point mutations in PYGL through WES, including a recurrent nonsense mutation c.280C $>$ T/p.R94X on exon 2 and a novel missense mutation c.1832C $>\mathrm{T} / \mathrm{p}$.A611V on exon 16, which can be classified as a likely pathogenic mutation. 


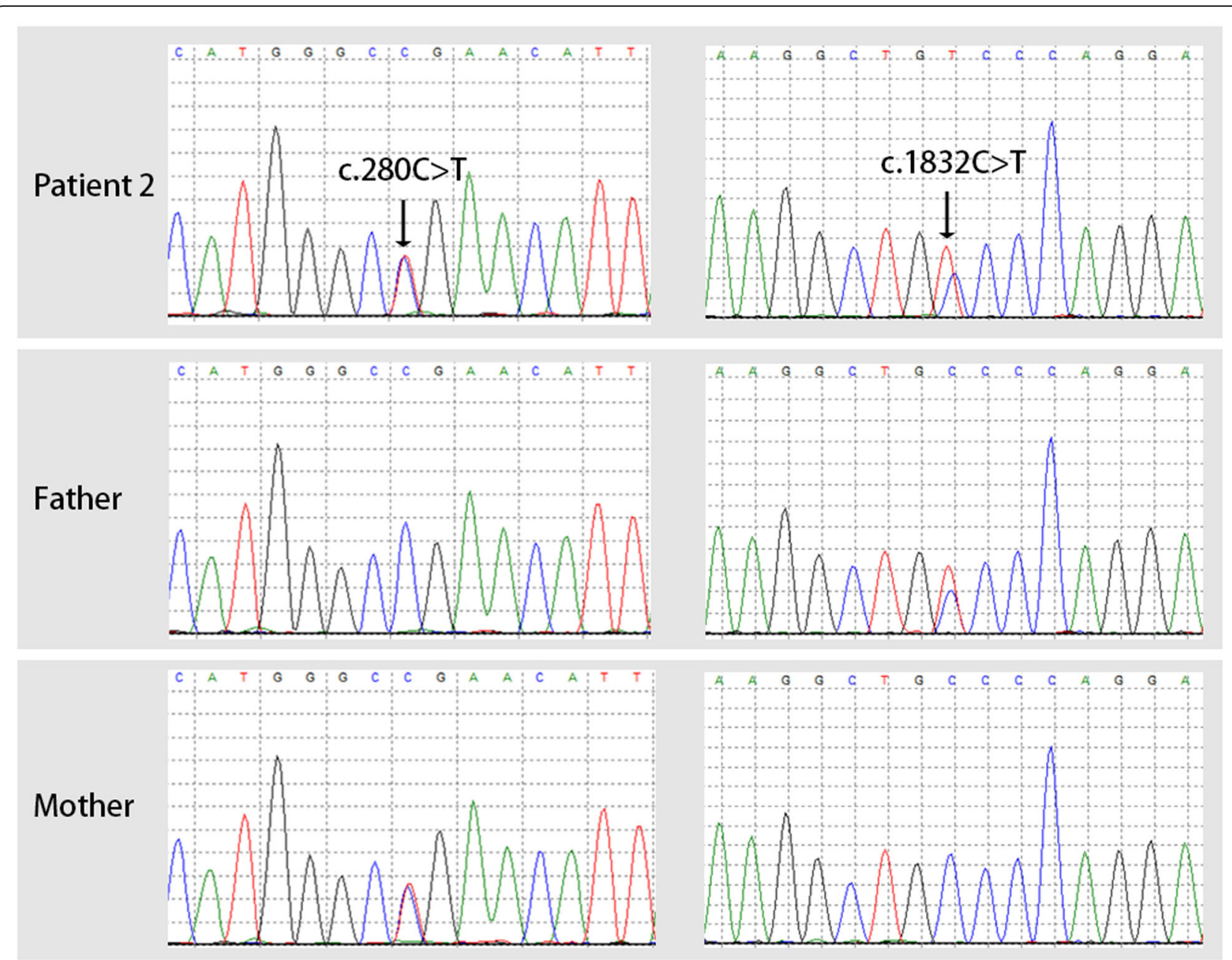

Fig. 2 Sanger sequencing validation of the PYGL gene mutations. The arrows show the compound heterozygous mutations of patient 2

Patients with GSD VI are generally normal at birth and receive attention during the infant stage due to short stature or hepatomegaly. Hepatomegaly is often regarded as the classic manifestation and presented in all of about 50 GSD VI pediatric patients described in literature [3, 8-12]. However, hepatomegaly was nearly absent in patient 1 in our study. We assume that some GSD VI patients may only manifest with short stature and increased liver transaminases without obvious hepatomegaly, which broadened the phenotype spectrum of GSD VI. Based on the nonspecific and variable nature of the disease, GSD VI patients is almost certainly underdiagnosed in the general population, leading to an underestimation of its prevalence. GSD VI can be under-diagnosed especially in China, because the ability for diagnosis of the disease varies greatly among the different geographical regions.

To date, missense/nonsense and splice site mutations have been the major PYGL mutation types in Human Gene Mutation Database, and gross deletion has rarely been reported. There are 20 exons and a total of $2544 \mathrm{bp}$ in the PYGL coding sequence. The liver phosphorylase consists of 847 amino acids and the structure of the protein has not been determined. The gross deletion of PYGL exons $14-17$ in patient 1 encodes 186 amino acids, which accounts for approximately $22 \%$ of the whole protein length. According to the ClinGen Sequence Variant Interpretation Working Group, removing $>10 \%$ of the protein product is more likely to have a loss of function effect compared to variants that remove $<10 \%$ of the protein [13]. However, the clinical course of patient 1 carrying the deletion were similar to the patient 2 carrying a nonsense mutation and a missense mutation. There is a high likelihood that this gross deletion may result in a stable expressed truncated protein. Mutations such as the gross in-frame deletion have implications for further interventions, such as gene therapy. For example, antisense oligonucleotide-mediated exon skipping is an emerging therapy for Duchenne muscular dystrophy patients. Exon skipping can restore the reading frame by removing the mutant exon and/or its flanking exon(s) from the pre- 
mRNA, leading to the expression of truncated but functional proteins [14]. The effect of this gross deletion on the liver phosphorylase requires further functional assays using either RNA or mutant protein analysis. RT-PCR and western blotting could not be conducted due to the unavailability of liver tissue.

Both of the two patients responded very well to frequent UCS treatment (4 times a day). They have achieved catch-up growth. For patient 1, all the biochemical parameters except for triglycerides were normal. Her laboratory test revealed mild hypertriglyceridemia. The hyperlipidemia is a result of both increased synthesis from excess of acetyl-coenzyme A via malonyl-coenzyme $\mathrm{A}$, and decreased serum lipid clearance. The hyperlipidemia responds to intensive dietary treatment, indicating hyperlipidemia is also a sign of altered glycogen metabolism and enhanced counterregulation hormone production associated with inadequate production of glucose from the liver. According to a retrospective study of six GSD VI patients treated with small, frequent meals as well as cornstarch in the evening, three younger patients showed persistent hyperglyceridemia and the youngest patient (2.5 years old) simultaneously showed obvious elevated transaminases $(\mathrm{ALT}=344 \mathrm{U} / \mathrm{L}, \mathrm{AST}=266 \mathrm{U} / \mathrm{L})$ even after 1.5 years' follow up [10]. A murine model of GSD VI revealed that elevated liver enzymes could increase the risk of liver damage, inflammation, and fibrosis [15]. We speculate that for younger patients, more frequent and intensive UCS treatment for the initial one year should be used to ensure the better metabolic control and catch-up growth.

In this study, we describe two unrelated patients of GSD VI and the clinical course. We report novel mutations (c.1832C > T/p.A611V and c.1621-258_2178-23del) and expand the PYGL mutation spectrum. Based on the results of our molecular investigation, the patients received precise diagnoses and efficient medical management.

\section{Supplementary information}

Supplementary information accompanies this paper at https://doi.org/10. 1186/s12881-020-01010-4.

Additional file 1 : Table S1. Primers used in PCR amplification (Fig. 1b), Table S2. Primers used in PCR amplification (Fig. 1c). Table S3. Primers used in fluorescent gap PCR amplification (Fig. 1d)

\section{Abbreviations}

WES: Whole exome sequencing; GSD: Glycogen storage diseases; UCS: Uncooked corn starch

\section{Acknowledgments}

We sincerely appreciate the families and clinicians for their participation in this project.

\section{Authors' contributions}

$W Q, J H$ and XG2 evaluated the clinical features and designed the study. YS annotated the WES data and called copy number variations through WES depth data. XL, XG1, YF analyzed the WES data of the patients. XL designed and performed the mutation verification assay. $\mathrm{XL}, \mathrm{JH}$ and $\mathrm{WQ}$ wrote the manuscript. All authors have read and approved the final manuscript.

\section{Funding}

This study was funded by Precision Medical Research of National Key Research and Development Program (2016YFC0905100 to WJQ and XFG), Shanghai Municipal Commission of Health and Family Planning (20184Y0073 to XML, 201940226 to WJQ). The funders had no role in the study design, data collection, data analysis, interpretation of results, and writing of the manuscript.

\section{Availability of data and materials}

The datasets used and analyzed during the current study are available from the corresponding author upon request. The phenotypes and mutations of the two GSD VI patients were submitted to Leiden Open Variation Database (https://databases.lovd.nl/shared/individuals) under accession number \#00295511 and \#00295495.

\section{Ethics approval and consent to participate}

This study was approved by Xinhua Hospital Ethics Committee Affiliated to Shanghai Jiao tong University School of Medicine (XHEC-D-2019-057). The control samples in Fig. 1 a and e were from volunteers. Written informed consent was obtained from parents of the patients and the controls.

\section{Consent for publication}

Written informed consent for publication of clinical details was obtained from the parents of participants.

\section{Competing interests}

The authors declare that they have no conflict of interest.

\section{Author details}

${ }^{1}$ Department of Pediatric Endocrinology/Genetics, Xinhua Hospital, Shanghai Jiao Tong University School of Medicine, Shanghai 200092, China. ${ }^{2}$ Shanghai Institute for Pediatric Research, Shanghai 200092, China. ${ }^{3}$ Shanghai Jiao Tong University School of Medicine, Shanghai 200025, China.

Received: 30 August 2019 Accepted: 24 March 2020

Published online: 08 April 2020

\section{References}

1. Roach PJ. Glycogen and its metabolism. Curr Mol Med. 2002;2(2):101-20.

2. Ozen H. Glycogen storage diseases: new perspectives. World J Gastroenterol. 2007;13(18):2541-53.

3. Burwinkel B, Bakker HD, Herschkovitz E, Moses SW, Shin YS, Kilimann MW. Mutations in the liver glycogen phosphorylase gene (PYGL) underlying glycogenosis type VI. Am J Hum Genet. 1998;62(4):785-91.

4. Richards S, Aziz N, Bale S, Bick D, Das S, Gastier-Foster J, et al. Standards and guidelines for the interpretation of sequence variants: a joint consensus recommendation of the American College of Medical Genetics and Genomics and the Association for Molecular Pathology. Genet Med. 2015; 17(5):405-24.

5. Kishnani PS, Goldstein J, Austin SL, Arn P, Bachrach B, Bali DS, et al. Diagnosis and management of glycogen storage diseases type $\mathrm{VI}$ and IX: a clinical practice resource of the American College of Medical Genetics and Genomics (ACMG). Genet Med. 2019;21(4):772-89.

6. Burda P, Hochuli M. Hepatic glycogen storage disorders: what have we learned in recent years? Curr Opin Clin Nutr Metab Care. 2015;18(4):415-21.

7. Wang J, Cui H, Lee NC, Hwu WL, Chien YH, Craigen WJ, et al. Clinical application of massively parallel sequencing in the molecular diagnosis of glycogen storage diseases of genetically heterogeneous origin. Genet Med. 2013;15(2):106-14

8. Beauchamp NJ, Taybert J, Champion MP, Layet V, Heinz-Erian P, Dalton A et al. High frequency of missense mutations in glycogen storage disease type VI. J Inherit Metab Dis. 2007;30(5):722-34.

9. Chang S, Rosenberg MJ, Morton H, Francomano CA, Biesecker LG. Identification of a mutation in liver glycogen phosphorylase in glycogen storage disease type VI. Hum Mol Genet. 1998;7(5):865-70.

10. Aeppli TR, Rymen D, Allegri G, Bode PK, Häberle J. Glycogen storage disease type VI: clinical course and molecular background. Eur J Pediatr. 2020;179(3): 405-13.

11. Roscher A, Patel J, Hewson S, Nagy L, Feigenbaum A, Kronick J, et al. The natural history of glycogen storage disease types $\mathrm{VI}$ and IX: long-term outcome from the largest metabolic center in Canada. Mol Genet Metab. 2014;113(3):171-6 
12. Tang NL, Hui J, Young E, Worthington V, To KF, Cheung KL, et al. A novel mutation (G233D) in the glycogen phosphorylase gene in a patient with hepatic glycogen storage disease and residual enzyme activity. Mol Genet Metab. 2003:79(2):142-5.

13. Abou Tayoun AN, Pesaran T, DiStefano MT, Oza A, Rehm HL, Biesecker LG, et al. Recommendations for interpreting the loss of function PVS1 ACMG/ AMP variant criterion. Hum Mutat. 2018;39(11):1517-24.

14. Echigoya Y, Lim KRQ, Trieu N, Bao B, Miskew Nichols B, Vila MC, et al. Quantitative antisense screening and optimization for exon 51 skipping in Duchenne muscular dystrophy. Mol Ther. 2017;25(11):2561-72.

15. Wilson LH, Cho J-H, Estrella A, Smyth JA, Wu R, Chengsupanimit T, et al. Liver glycogen phosphorylase deficiency leads to profibrogenic phenotype in a murine model of glycogen storage disease type VI. Hepatol Commun. 2019;3(11):1544-55.

\section{Publisher's Note}

Springer Nature remains neutral with regard to jurisdictional claims in published maps and institutional affiliations.

Ready to submit your research? Choose BMC and benefit from:

- fast, convenient online submission

- thorough peer review by experienced researchers in your field

- rapid publication on acceptance

- support for research data, including large and complex data types

- gold Open Access which fosters wider collaboration and increased citations

- maximum visibility for your research: over $100 \mathrm{M}$ website views per year

At $\mathrm{BMC}$, research is always in progress.

Learn more biomedcentral.com/submissions 